\title{
Nanoelectrochemistry and Nanoelectrics at Electrode/Electrolyte Interface
}

\author{
Zhuangqun Huang, Peter De Wolf, and Bede Pittenger
}

Bruker Nano Surfaces, Santa Barbara, CA, USA

In situ electrical and electrochemical (EC) studies with nanometer resolution at electrified solidelectrolyte interfaces are critical for understanding the complex fundamental mechanisms in energy storage and conversion systems, biological applications, and sensors. In these studies, atomic force microscopy (AFM) has been widely adopted. For example, PeakForce EC-AFM allows for topographic and nanomechanical imaging during EC reactions, e.g. visualization of in situ SEI evolution of a Li-ion battery anode [1]. Integration with scanning electrochemical microscopy (SECM) enables the simultaneous extended capture of local EC and conductivity information. For instance, the recently developed PeakForce SECM using a nanoelectrode probe with an active conical Pt tip apex of $\sim 25 \mathrm{~nm}$ in radius [2] has been used to characterize the activities of Pt catalysts on a hydrogen evolution photoanode. [3]

Recently, we have developed Force Volume SECM (FV-SECM) providing improved electrochemical kinetic quantification, 3-D nano-EC, and nanomechanics. In this mode, the AFM nanoelectrode probe is moved from pixel to pixel in a fast force volume mapping (FFV) method, providing a force-distance spectrum in each pixel. During each capture of the force spectrum, each probe ramping cycle provides an SECM probe approach curve (PAC), as shown in Figure 1(a). This method breaks the limitation of conventional SECM methods from which only few PACs are inefficiently captured from a single or very few point locations. For kinetic studies, in literature [4], researchers often quantify reaction kinetics from the two-dimensional (2D) SECM current image with the aid of a collection of simulated PACs. FV-SECM bypasses this PAC simulation and can greatly improve the quantification accuracy. FVSECM also allows plotting the SECM current images at different tip-sample distances with nm step accuracy. In addition, during each force-distance cycle of FV-SECM, the probe can be held on the surface for a pre-defined time at a fixed force or $\mathrm{Z}$ position. When the probe is in the 'hold' stage, spectra of surface response are collected by varying one of the operating conditions. The built-in ramp and hold function also makes nanocontact for EC studies possible, e.g. local surface EC potential measurement. [5-6]

Nanoelectric techniques have been widely used in air. However, many applications require performing such studies in electrolyte solutions. Taking Li ion battery as an example, the SEI layer is desired to be ionically conductive, electrically insulating, and mechanically elastic and robust. Probing electrical properties in situ during the SEI formation is valuable for practical device development. In a biological system, besides the bioelectricity, a living organism is generally an electromechanical system, that is, mechanical responses results from electrical stimulation, or vice versus. However, all these phenomena occur in a biological environment, that is, in a solution with an electrolyte concentration of $\sim 150 \mathrm{mM}$ and at a certain $\mathrm{pH}$. For nanoparticle catalysis, the interfacial conductivity is a critical factor to determine the device performance and a direct measurement in electrolyte is more helpful than through conventional indirect EC methods with simulation. All these cases require nanoelectrical measurement in liquid, which however, is not feasible to implement today. This is due to known challenges. First, the measurement generally requires a full immersion of both a conductive AFM probe/nanoelectrode tip and the sample in electrolyte. However, to fulfill this scheme without electrical shorting, liquid spilling or 
chemical corrosion is challenging. Second, high signal-to-noise ratio requires a carefully-designed insulating scheme to minimize undesired contribution from parasitic electrochemistry and stray capacitance. By the development and the use of the nanoelectrode tip, we have overcome these technical hurdles and developed a suite of solutions to conduct multi-dimensional nanoelectrical measurements in liquid, including piezo response, conductivity, IV spectra and Kelvin Probe mapping. The FFV method is also implemented for capture multi-dimensional datacube in liquid whereby mechanical and other spectra are collected for each pixel. As shown in Figure 1(b), we have employed the FFV-Piezo Force Microscopic (FFV-PFM) methods to study piezoelectric materials, e.g. PIMNT and PPLN, where pixelwise bias-sweeping spectrum (switching loops) reveals dynamic evolution of domain walls, and frequency-based spectra allow studying the contact resonance properties. [7]

In this work, I will introduce these recently-developed or newly-invented, unique techniques with a variety of examples in energy, biological, and semiconductor applications, such as Li-ion batteries, solar water splitting devices, electrocatalysis, and bio-electromechanical materials.

\section{References:}

[1] Kuma et al., ACS Appl. Mater. Interfaces 9 (2017), p. 28406.

[2] Nellist et al., Nanotechnology 28 (2017), p. 095711.

[3] Jiang et al., ChemSusChem 2017, DOI: 10.1002/cssc.201700893.

[4] Macpherson \& Unwin et al., Angew. Chem. Int. Ed. 51 (2012), p. 7002.

[5] Nellist et al., Nature Energy, 3 (2018), 46.

[6] Toma, Nature Energy 3 (2018), p. 6.

[7] Huang et al., Microscopy Today 6 (2017), p. 18.

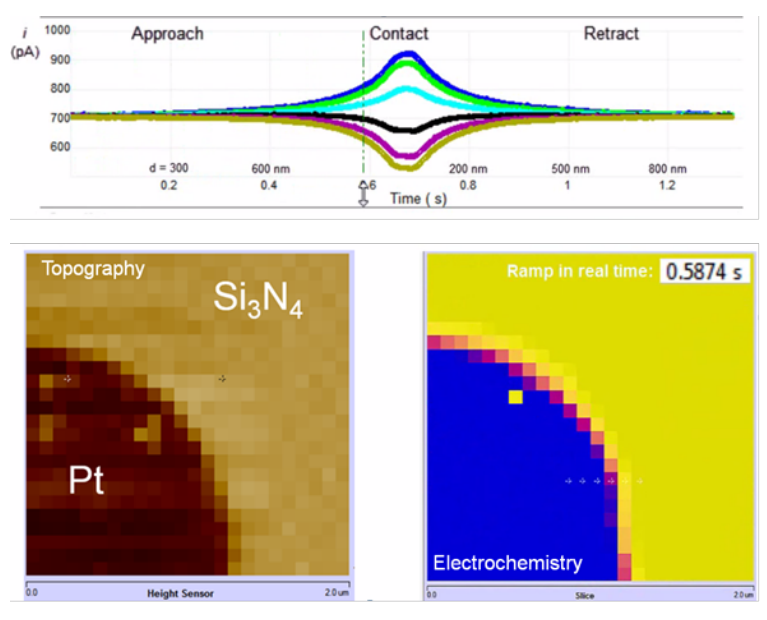

(a)

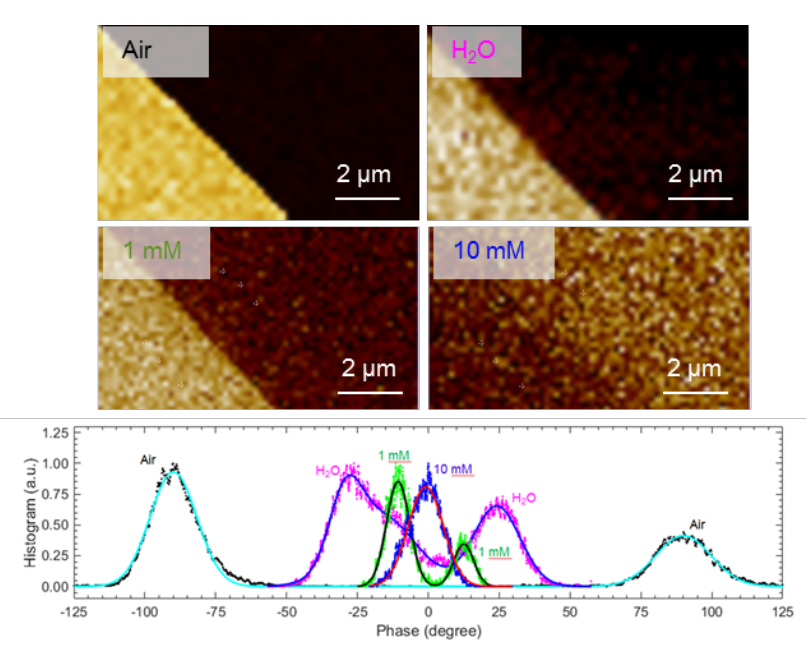

(b)

Figure 1. (a). FV-SECM of an $\mathrm{Pt} / \mathrm{Si}_{3} \mathrm{~N}_{4}$ pattern electrode: top plots show PCAs at different locations with different EC activities and bottom images show topography and electrochemistry at the slice indicated at the top plot. (b). FFV-PFM in liquid: top and bottom images show PFM images of PPLN and phase plots, respectively, at contact resonance in different environments as indicated. 